\title{
Ecografía renal con Doppler en el diagnóstico de pielonefritis aguda en niños
}

\author{
FELIPE CAVAGNARO S. ${ }^{1}$, LUISA SCHONHAUT B ${ }^{1}$, \\ BÁRBARA MORALES K. ${ }^{2}$, ANÍBAL ESPINOZA G. ${ }^{2}$ \\ 1. Departamento de Pediatría, Facultad de Medicina, Clínica Alemana-Universidad del Desarrollo. \\ 2. Departamento de Imagenología, Facultad de Medicina, Clínica Alemana-Universidad del Desarrollo.
}

\begin{abstract}
Renal ultrasound with Doppler in the diagnosis of acute pyelonephritis in children

Objective: This study evaluates the diagnostic value of Renal Doppler Ultrasound (RDU) in the diagnosis of acute renal involvement among children admitted for a first febrile urinary tract infection (UTI), compared to a Renal Scintigraphy (RS). Methodology: All children admitted during 2009 for a first febrile UTI and positive urinary culture were prospectively studied. All received a RDU and RS with Tc99di-mercaptosuccinic acid during the first 72 hours of hospitalization. Image evaluation was performed blindly. RS was considered to be the gold standard to identify renal involvement. Results: The protocol was completed in 54 children (90\%). RDU was altered in $36(66 \%)$, and RS in $42(78 \%)$ of children. Both exams coincided in $78 \%$ of the children, and in $81 \%$ of the kidneys. Sensitivity and specificity of RDU for these patients was $78,6 \%$ and $75 \%(72 \%$ and $89,6 \%$ per kidney), respectively. Negative predictive value was $50 \%$. RDU detected significantly fewer defects in the left kidney, $(p=0,0026)$, especially if the defect was localized in the upper pole, and in absence of other associated scintigraphic abnormalities $(\mathrm{p}=0,0001)$. Children with scintigraphic renal involvement showed significant more alterations during the acute phase. Conclusions: RDU is sensitive and specific to detect renal involvement in a child admitted for a febrile UTI, but has a low negative predictive value. Therefore, a negative ultrasound in a patient with inflammatory signs should be confirmed through a scintigraphy, since there are certain sites that are difficult to localize in a sonogram, such as the left superior pole.

(Key words: Renal scintigraphy, RDU, pyelonephritis, urinary infection).

Rev Chil Pediatr 2011; 82 (5): 402-409
\end{abstract}

\section{RESUMEN}

Introducción: El objetivo del presente estudio fue evaluar las propiedades diagnósticas de la ecografía renal con Doppler (ERD) en la identificación del compromiso renal agudo, en una cohorte de niños hospitalizados con primera infección urinaria (ITU) febril y compararlas con el cintigrama renal estático (CR). Metodología: Durante el año 2009, en forma prospectiva, se estudiaron todos los niños hospitalizados con primera ITU

Trabajo recibido el 10 de abril de 2011, devuelto para corregir el 06 de junio de 2011, segunda versión el 28 de junio de 2011 , aceptado para publicación el 04 de agosto de 2011.

Correspondencia a:

Felipe Cavagnaro S.

E-mail: fcavagnaro@alemana.cl 
febril y con urocultivo positivo. En todos ellos se realizó una ERD y un CR con ácido dimercapto succínico marcado con tecnecio 99, dentro de las primeras 72 horas de hospitalización. Los estudios de imágenes fueron realizados en forma ciega, por operadores independientes. El CR se consideró como gold standard para la identificación de compromiso renal. Resultados: En 54 niños (90\% de los elegibles) hospitalizados por ITU febril se cumplió el protocolo de estudio imageneológico. La ERD estuvo alterada en $36(66 \%)$ y el CR en $42(78 \%)$ de los niños. Ambos exámenes fueron coincidentes en $78 \%$ de los niños y en $81 \%$ de los riñones estudiados. La sensibilidad y especificidad del la ERD para estos pacientes fueron de 78,6\% y $75 \%$ ( $72 \%$ y $89,6 \%$ al analizarlo por unida renal), respectivamente, y el valor predictivo negativo de 50\%. La ERD pesquisó significativamente menos defectos cuando éstos eran exclusivos del riñón izquierdo $(p=0,0026)$, especialmente si el defecto se localizó en el polo superior y en ausencia de otras alteraciones cintigráficas asociadas ( $p$ $=0,0001)$. Los niños con compromiso renal cintigráfico tenían significativamente más alterados los reactantes de fase aguda. Conclusiones: La ERD en un niño hospitalizado por una ITU febril tiene alta sensibilidad y especificidad para pesquisar el compromiso renal, pero un bajo valor predictivo negativo; por lo tanto, si la ERD es negativa y el paciente presenta parámetros inflamatorios alterados, el compromiso renal debe ser confirmado por un CR, ya que existe evidencia de ciertas localizaciones más difíciles de identificar por la ERD, como aquellas que afectan el polo superior izquierdo.

(Palabras clave: Cintigrama renal, ecografía renal con doppler, pielonefritis, infección urinaria).

Rev Chil Pediatr 2011; 82 (5): 402-409

\section{Introducción}

La Infección del Tracto Urinario (ITU) es una de las principales causas de síndrome febril sin foco en niños menores de 5 años y la segunda infección bacteriana más frecuente en pediatría, después de las infecciones respiratorias. Esta patología conlleva una considerable responsabilidad médica en relación a su certero y oportuno diagnóstico, adecuado estudio y correcto tratamiento ${ }^{1}$.

En forma clásica, las ITUs se diferencian clínicamente entre infecciones altas o pielonefritis aguda (PNA), y bajas o cistitis. Esta separación arbitraria es extremadamente importante pues las primeras se pueden asociar a daño renal permanente, al tiempo que se relacionan con mayor frecuencia a malformaciones nefrourológicas y reflujo vésico ureteral (RVU) ${ }^{1,2}$.

Si bien sugerentes y sensibles, el cuadro clínico y los exámenes de laboratorio en lactantes y preescolares son poco específicos en relación a la ubicación de la ITU. Por este motivo ha sido relevante incorporar estudios de imágenes como elementos centrales en la evaluación de pacientes pediátricos con este tipo de patologíá ${ }^{1,3,4}$.

Entre los estudios actualmente disponibles, el cintigrama renal (CR) con ácido dimercapto succínico marcado con tecnecio $99\left(\mathrm{Tc}^{99} \mathrm{DM}\right.$ -
SA) representa una herramienta diagnóstica fundamental ${ }^{4}$. No obstante, el CR presenta como desventajas el no diferenciar el compromiso cortical agudo del cicatricial y no está ampliamente disponible en nuestro país; además, requiere de un acceso venoso, expone al paciente y familiares a mínima irradiación (sólo relevante en caso de embarazadas) y puede, muy ocasionalmente, requerir sedación en su realización ${ }^{4-6}$.

La ecografía renal simple se ha planteado como una alternativa diagnóstica accesible y de bajo costo, al tiempo que evalúa concomitantemente posibles malformaciones de vía urinaria. El problema de esta técnica, operador dependiente, es su baja sensibilidad en identificar la ubicación de la ITU ${ }^{7,8}$, por lo que una ecografía normal no permite descartar una pielonefritis con seguridad. La incorporación del efecto Doppler a la ecografía ha sido promisorio como herramienta diagnóstica, debido a su precisión para identificar áreas con compromiso vascular focal o difuso, sugerentes de la localización y nivel de la inflamación renal, en un cuadro de ITU.

Si bien hay bastante evidencia internacional que considera la Ecografía renal con Doppler (ERD) como válida y recomendable ${ }^{7-9}$, no existe información sobre esta alternativa en la literatura nacional. 
El objetivo del presente estudio fue evaluar las propiedades diagnósticas de la ERD en la identificación del compromiso renal agudo, en una cohorte de niños hospitalizados con primera ITU febril y compararlas con el CR como gold standard.

\section{Pacientes y Método}

Estudio prospectivo, observacional y analítico. Entre el $1^{\circ}$ de enero y el 31 de diciembre de 2009 se reclutaron todos los niños menores de 15 años, hospitalizados con primer episodio de ITU febril, en el Servicio de Pediatría de una clínica que atiende niños de nivel socio económico medio alto, de la Región Metropolitana.

Se consideró ITU febril la existencia de un cuadro clínico caracterizado por fiebre, orina completa alterada y urocultivo positivo, en ausencia de otro foco infeccioso evidente.

La decisión de hospitalización de los pacientes fue discrecional del médico tratante derivador o del médico que evaluó al paciente en el Servicio de Urgencia de nuestra institución, guiados por las normas publicadas al respecto $^{1,10}$.

La muestra para urocultivo fue obtenida por recolector, cateterización vesical o captación de orina de segundo chorro, según la edad y cooperación del paciente.

Se excluyeron aquellos niños con urocultivo negativo, antecedentes de ITUs previas, daño renal agudo o crónico previo, malformación de vía urinaria obstructiva, quistes o hipo/ displasia renal, vejiga neurogénica y rechazo de los padres a participar.

De acuerdo a un protocolo establecido, los niños fueron estudiados a su ingreso hospitalario con, al menos, los siguientes exámenes de laboratorio: hemograma con velocidad de eritrosedimentación (VHS) y proteína $\mathrm{C}$ reactiva (PCR). El estudio de imágenes consistente en ERD y CR, fue realizado dentro de las siguientes 48 horas de hospitalización.

Las ERD fueron efectuadas e interpretadas por 3 radiólogos pediátricos con equipo Phillips, modelo iU22, transductores de frecuencia múltiple, convexo 9-4 Mhz y lineal 12-5 Mhz. Se realizaron imágenes longitudinales y transversales a los riñones con abordaje lateral y prono. Se usaron criterios morfológicos sumados a la evaluación de mapeo renal con Doppler color para diagnóstico de $\mathrm{PNA}^{8}$. Todas las imágenes fueron posteriormente reevaluadas por un radiólogo pediátrico que consensuó el diagnóstico. El CR fue realizado 2 a 3 horas después de la administración de una dosis i.v. de $T \mathrm{~T}^{99} \mathrm{DMSA}$, obteniéndose imágenes de ambos riñones en proyección anterior, posterior y ambas oblicuas posteriores utilizando una gamma cámara modelo Millenium (GE), colimador pinhole, matriz $256 \times 256$, zoom 2.0 y 300000 cuentas por imagen. Se efectuó análisis visual de las imágenes y cuantificación de la función diferencial. Los criterios de interpretación se basaron en las últimas guías norteamericanas $^{11}$ y europeas ${ }^{12}$ de Medicina Nuclear Pediátrica.

Los evaluadores fueron ciegos al resultado del otro estudio de imagen.

\section{Definición de exámenes}

Urocultivo: se consideró el urocultivo alterado cuando el recuento de unidades formadoras de colonias fue $\geq$ a $100000 / \mathrm{mm}^{3}$ en muestra tomada por recolector o "segundo chorro", $\mathrm{o} \geq 10000 / \mathrm{mm}^{3}$ en muestra obtenida por cateterización vesical transitoria ${ }^{1,13}$.

Análisis de orina: presencia de leucocitos/ piocitos $\geq 6$ por campo de mayor aumento, test de nitritos $(+)$, bacteriuria $(+)$ o cilindros piocitarios.

Criterios ERD de PNA: incremento de tamaño renal focal o generalizado; alteración de la ecogenicidad del parénquima (áreas hipo o hiperecogénicas); pérdida de la diferenciación córtico-medular, y engrosamiento del urotelio en la pelvis y/o del uréter ${ }^{14}$. Además del hallazgo de una zona focal hipovascular en el mapeo renal con Doppler color.

Criterios cintigrafía renal para PNA: áreas focales corticales hipocaptantes, defectos corticales, aumento de volumen localizado en un área afectada y aumento de volumen difuso de un riñón con múltiples defectos ${ }^{11,12,14}$. Se rotuló el número de alteraciones corticales y su localización por unidad renal y se consideró función diferencial relativa normal entre el rango $45-55 \%$. 


\section{Análisis estadístico}

Se realizó un análisis descriptivo a través de frecuencias para variables categóricas y estadísticas de tendencia central para las variables continuas. Aquellas variables que no siguieron una distribución normal, se describieron a través de medianas y rangos. Los parámetros clínicos y de laboratorio fueron comparados para los grupos $\mathrm{CR}+\mathrm{y} \mathrm{CR}$ - a través de la prueba de Mann-Whitney. Se consideraron significancias estadísticas para un valor de $\mathrm{p}<0,05$.

Para los distintos parámetros de laboratorio y para el ERD se calculó la sensibilidad, especificidad, valores predictivos y razón de verosimilitud (LR por likelihood ratio), con sus respectivos intervalos de confianza, considerando como gold standard la CR, para el diagnóstico de compromiso renal.

\section{Resultados}

Durante el año de estudio, se hospitalizaron 74 niños con diagnóstico de ITU febril. Catorce fueron excluidos debido a historia de ITU previas y/ o antecedente de malformación obstructiva de la vía urinaria. De los 60 niños elegibles para el estudio, en $6(10 \%)$ no se cumplió el protocolo de estudio imagenológico en el período establecido, por lo que fueron excluidos y la muestra final a analizar quedó conformada por 54 niños.

La edad promedio de los pacientes fue de 14 meses (mediana de 5 meses, rango de 10 días a 12 años, $63 \%<1$ año), $30(55 \%)$ eran de sexo femenino. Entre los síntomas clínicos destaca que la totalidad de los niños tenía historia de fiebre (promedio y mediana de 2 días, rango de 2 horas hasta 7 días), además de un análisis de orina positivo. En todos ellos se demostró un urocultivo positivo a germen único (84\% Escherichia coli). De los parámetros de laboratorio medidos, $76 \%$ tenía leucocitosis $>15000 \times \mathrm{xm}^{3}$ (promedio 18813 , rango 7900 a $\left.35100 \times \mathrm{mm}^{3}\right), 88 \%$ con VHS $>30$ $\mathrm{mm} / \mathrm{h}$ (promedio 61, rango 26 a $115 \mathrm{~mm} / \mathrm{h}$ ) y $76 \%$ con PCR $>5 \mathrm{mg} / \mathrm{dl}$ (promedio 10,7, rango $0,5$ a $28,2 \mathrm{mg} / \mathrm{dl})$.

En relación a los estudios de imágenes, la ERD estuvo alterada en $36(66 \%)$ de los ni- ños, correspondiendo a 42 unidades renales, y el CR en $42(78 \%)$ de los niños y 50 unidades renales. De estas últimas, 29 (58\%) correspondieron al riñón izquierdo y de ellos fueron 8 bilaterales. Los principales hallazgos cintigráficos se muestran en la tabla $1 \mathrm{y}$ en la figura 1 . La ERD fue coincidente con el CR en 42 niños (78\%) y en 88 riñones (81\%).

Dependiendo de la presencia de compromiso renal demostrado por $\mathrm{CR}$, los niños fueron catalogados en Grupo CR+ y Grupo CR-.

En la tabla 2 se presentan los datos clínicos y de laboratorio según esta clasificación. Al comparar ambos grupos destaca que no hubo diferencias en los parámetros clínicos, pero sí en los estudios de laboratorio. El grupo de niños con $\mathrm{CR}+$ tenía significativamente más alterados los reactantes de fase aguda como PCR, VHS y leucocitosis.

En la tabla 3 se muestra la sensibilidad, especificidad y valores predictivos de los diferentes parámetros de laboratorio y de la ERD en la detección de compromiso renal evidenciado por el CR. Destaca que, con los puntos de corte considerados, todos los parámetros analizados tenían una sensibilidad mayor al $75 \%$, pero con valores de especificidad bajos, salvo la ERD cuya especificidad fue significativamente superior, y con un valor predictivo positivo mayor a $90 \%$.

Al observar separadamente los resultados por unidades renales (tabla 4), destaca que la ERD tiene una moderada sensibilidad pero

Tabla 1. Tipo de compromiso cintigráfico en $\mathbf{5 0}$ unidades renales

\begin{tabular}{|lrr|}
\hline Compromiso cintigráfico & n & \% \\
\hline Defecto de polo superior & 28 & 56 \\
\hline Defecto de polo inferior & 1 & 2 \\
\hline Defectos bipolares & 6 & 12 \\
\hline Defecto polar + otro & 2 & 4 \\
\hline Defecto bipolar + otro & 5 & 10 \\
\hline Defectos no polares & 5 & 10 \\
\hline Defectos multifocales & 3 & 6 \\
\hline Riñón globuloso afectado & 15 & 30 \\
\hline Defectos únicos & 34 & 68 \\
\hline Defectos múltiples & 16 & 32 \\
\hline
\end{tabular}



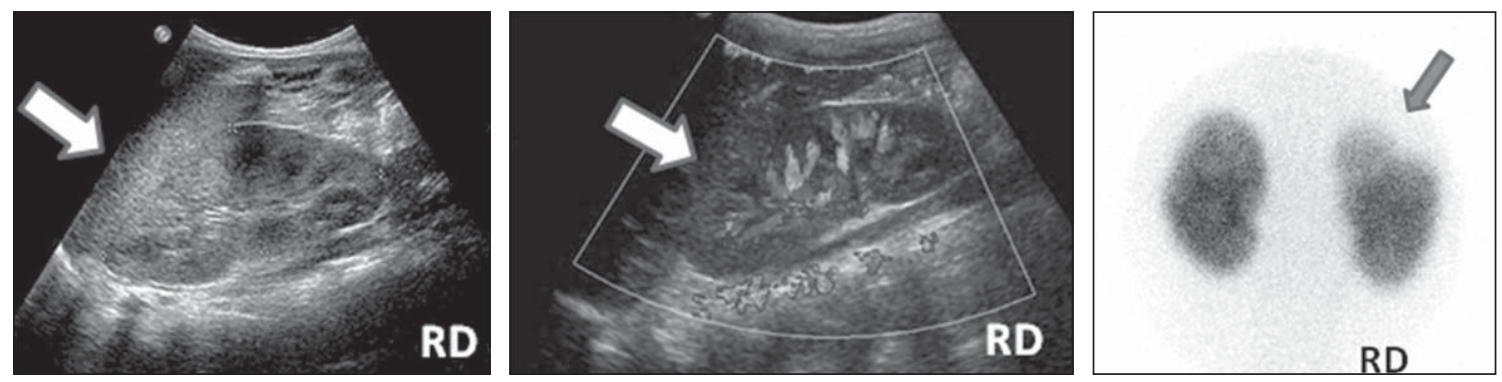

Figura 1. Pielonefritis aguda con compromiso de polo superior de riñón derecho en un niño, visualizado por ecografía renal simple, ecografía renal con doppler y Tc ${ }^{99}$ DMSA cintigrama renal. A. En la Ecografía Renal Simple se puede observar un aumento de la ecogenicidad y pérdida de la diferenciación corticomedular en el polo superior del riñón derecho. B. En la Ecografía Renal con Doppler se puede apreciar además que en la zona de mayor ecogenicidad existe una disminución del flujo sanguíneo. C. En la Cintigrafía Renal destaca un defecto cortical del polo superior del riñón derecho.

Tabla 2. Resultados obtenidos en los diferentes parámetros clínicos y de laboratorio estudiados según cintigrama renal

\begin{tabular}{|llll|}
\hline Parámetro & CR (+) & CR (-) & P \\
\hline $\mathrm{n}$ & 42 & 12 & $\mathrm{NS}$ \\
\hline Edad en meses, promedio (rango) & $14,8(0-60)$ & $13,9(1-144)$ & $\mathrm{NS}$ \\
\hline Sexo masculino $\mathrm{n}(\%)$ & $22(52)$ & $4(33)$ & $\mathrm{NS}$ \\
\hline Fiebre previo hospitalización: días promedio (rango) & $2,1(0-7)$ & $1,9(0-5)$ & $\mathrm{NS}$ \\
\hline Urocultivo (+) a E. coli $\mathrm{n}(\%)$ & $25(59)$ & $10(83)$ & $\mathrm{NS}$ \\
\hline Leucocitos x mm ${ }^{3}$, promedio \pm DS & $20063 \pm 6172$ & $14858 \pm 5304$ & $\mathrm{p} 0,012^{* *}$ \\
\hline $\mathrm{VHS}, \mathrm{mm} / \mathrm{h}$, promedio \pm DS & $68 \pm 29$ & $38 \pm 16$ & $\mathrm{p} 0,015^{* *}$ \\
\hline $\mathrm{PCR}, \mathrm{mg} / \mathrm{dl}$, mediana y rango* & $12(1-28)$ & $4(1-27)$ & $\mathrm{p} 0,018^{* *}$ \\
\hline
\end{tabular}

*Dado que la distribución de los valores de PCR no siguieron una curva normal, se presentan los valores de mediana y rango. **Estadísticamente significativo (Mann Whitney). CR: cintigrama renal, VHS: velocidad de eritrosedimentación, PCR: proteína C reactiva, DS: desviación standard.

Tabla 3. Rendimiento relativo de los diferentes parámetros estudiados para el diagnóstico de pielonefritis aguda*

\begin{tabular}{|c|c|c|c|c|c|c|c|c|}
\hline \multirow[b]{2}{*}{ Sensibilidad } & \multicolumn{2}{|c|}{ VHS > $30 \mathrm{~mm} / \mathrm{h}$} & \multicolumn{2}{|c|}{ Leucocitos $>15000 \times \mathrm{mm}^{3}$} & \multicolumn{2}{|c|}{ PCR $>5 \mathrm{mg} / \mathrm{dl}$} & \multicolumn{2}{|r|}{ ERD } \\
\hline & 96 & $(I C 86,3-100)$ & 81,6 & (IC 67,9 - 95,2) & 78,6 & $($ IC $65-92,2)$ & 78,6 & (IC $65-92,2)$ \\
\hline Especificidad & 28,6 & $($ IC $0-69)$ & 41,7 & $(I C 9,6-73,7)$ & 58,3 & (IC 26,3-90,4) & 75 & $($ IC $46,3-100)$ \\
\hline VPP & 82,8 & $($ IC 67,3 - 98,2) & 81,6 & $($ IC $67,9-95,2)$ & 86,8 & $($ IC $74,8-98,1)$ & 91,7 & $($ IC 81,3 - 100) \\
\hline VPN & 66,7 & (IC 0 - 100) & 41,7 & (IC 9,6 - 73,7) & 43,8 & (IC 16,3 - 71,2) & 50 & (IC 24,1 - 75,9) \\
\hline
\end{tabular}

*(Considerando el CR como gold standard). VPP: valor predictivo positivo; VPN: valor predictivo negativo; IC: intervalo de confianza. VHS: velocidad de eritrosedimentación, PCR: proteína C reactiva, ERD: ecografía renal con doppler.

Tabla 4. Resultados comparativos del cintigrama renal con la ecografía renal con Doppler en 54 pacientes y 108 riñones estudiados

\begin{tabular}{|llccccccccc|}
\hline ERD & & $\begin{array}{c}\text { CR (+) } \\
\mathbf{n}\end{array}$ & $\begin{array}{c}\text { CR (-) } \\
\mathbf{n}\end{array}$ & Total & $\begin{array}{c}\text { S } \\
\mathbf{\%}\end{array}$ & $\begin{array}{c}\text { E } \\
\mathbf{\%}\end{array}$ & $\begin{array}{c}\text { VPP } \\
\mathbf{\%}\end{array}$ & $\begin{array}{c}\text { VPN } \\
\mathbf{\%}\end{array}$ & LR(+) & LR (-) \\
Por paciente & Alterado & 33 & 3 & 36 & 78,6 & 75 & 91,7 & 50 & 3,14 & 0,29 \\
& Normal & 9 & 9 & 18 & & & & & & \\
$\begin{array}{l}\text { Por unidad } \\
\text { renal }\end{array}$ & Alterado & 36 & 6 & 42 & 72 & 89,7 & 85,7 & 78,8 & 6,96 & 0,31 \\
\hline
\end{tabular}

CR: cintigrama renal, ERD: ecografía renal con Doppler; S: sensibilidad, E: especificidad, VPP: valor predictivo positivo, VPN: valor predictivo negativo. LR razón de verosimilitud. 
muy buena especificidad y valores predictivos. La razón de verosimilitud (LR) no modifica significativamente la probabilidad pre-test de presentar compromiso renal, tanto en el análisis por paciente como por unidad renal.

La ERD pesquisó significativamente menos defectos cuando éstos eran exclusivos del riñón izquierdo $(p=0,0026)$, especialmente si el defecto se localizó en el polo superior de dicho riñón, sin otras alteraciones cintigráficas asociadas $(p=0,0001)$. Los 3 niños que aparecieron con compromiso renal en la ERD y tenían CR negativo, presentaron dilatación del seno renal como único hallazgo ecográfico.

\section{Discusión}

La asociación de PNA en edad pediátrica con posibles secuelas renales e hipertensión, parecen indicar la conveniencia de ubicar el sitio de inflamación asociado a estas infecciones, lo que determina el estudio, tratamiento y seguimiento.

Nuestra muestra de niños hospitalizados con su primera ITU febril, representa un universo seleccionado en términos de gravedad y/o riesgo clínico, lo que podría explicar la alta frecuencia de parámetros de laboratorio alterados y la elevada tasa de compromiso renal evidenciado en la cintigrafía (78\%), similar a otros estudios, tanto nacionales ${ }^{7}$ como extranjeros $^{15,16}$.

Al separar los niños de acuerdo a la presencia de compromiso renal cintigráfico, destaca que no encontramos diferencias en las variables clínicas (edad, género, días de fiebre), pero sí en los parámetros de laboratorio, los que estaban significativamente más alterados en el grupo de niños con compromiso renal. Estos parámetros, como ya ha sido descrito previamente, mostraron alta sensibilidad pero baja especificidad ${ }^{17-19}$, puesto que pueden elevarse frente a diversos cuadros infecciosos que podrían haber coexistido en los niños hospitalizados ${ }^{7}$.

Considerando que la clínica y los parámetros de laboratorio son inespecíficos como indicadores de compromiso renal en un cuadro de ITU, se han ido incorporando técnicas de imágenes en los protocolos de estudio de los niños ITU febril. Entre estos el CR con $\mathrm{Tc}^{99}$ DMSA es considerado actualmente como método de referencia, aun considerando sus desventajas $^{6,14,16}$.

La ecografía renal simple, que inicialmente se planteó como método alternativo, carece de precisión diagnóstica, aspecto que se ha perfeccionado agregando el efecto Doppler co$\operatorname{lor}^{5,6}$.

Al comparar la ERD con el CR, encontramos coincidencia entre ambas técnicas en $78 \%$ de los pacientes, correspondiendo a $81 \%$ de las unidades renales. La ERD demostró elevados valores de sensibilidad y especificidad, tanto en el análisis por unidad renal (72 y 89,6\%, respectivamente), como en el análisis por paciente $(78,6$ y $75 \%$, respectivamente), que es en definitiva lo que cuenta a la hora de tomar una decisión clínica. Los elevados valores de sensibilidad y valor predictivo positivo, comparables con estudios realizados en distintos países ${ }^{5,8,9,14}$ podrían estar afectados por la alta prevalencia de compromiso renal en la población estudiada. La razón de verosimilitud (LR) no modificó significativamente la probabilidad pre-test de presentar compromiso renal.

El 15\% de falsos negativos en la ERD correspondió en su gran mayoría a lesiones multifocales y/o del polo superior del riñón izquierdo, en ausencia de otro compromiso renal asociado. Distintas publicaciones reportan limitaciones técnicas de la ERD, como transductores de frecuencia inadecuada, artefactos, poca cooperación del paciente ${ }^{7,8}$ y obesidad ${ }^{9}$, variables no evaluadas en nuestro estudio. Por otro lado, se ha descrito que la ausencia de la ventana acústica hepática y la presencia de gas intestinal dificultan la visualización adecuada del flujo sanguíneo en la porción superior del riñón izquierdo ${ }^{5,8}$, lo que concuerda con nuestros hallazgos.

Majd y cols, plantean que algunos falsos negativos pueden deberse a una obstrucción venosa parcial causado por el edema renal en la fase aguda de PNA, lo que produce un aumento de volumen y enlentecimiento del flujo sanguíneo en la parte inflamada ${ }^{4}$.

En cuanto al 5\% de falsos positivos, deberíamos preguntarnos si se deben a un error de interpretación de las imágenes, ya que son 
operador dependientes, o bien a una limitación de la CR como gold Standard, cuyo uso en la fase aguda de una PNA es discutido ${ }^{20,21}$, especialmente en niños muy pequeños ${ }^{6}$. Estudios en modelos animales, en los que se comparan los hallazgos cintigráficos con la confirmación anátomo-patológica de la infección del tejido renal, han mostrado, en promedio, una sensibilidad de un $86 \%$ y una especificidad de un $91 \%{ }^{22}$. Entre las lesiones renales que habitualmente no son pesquisadas cintigráficamente destacan las extracorticales (ej, pielitis), y las corticales muy pequeñas, lesiones que probablemente tampoco serían detectadas con otras técnicas de imágenes menos sensibles ${ }^{8,14}$.

$\mathrm{Al}$ evaluar los hallazgos ecográficos en los falsos positivos, todos correspondieron exclusivamente a dilatación leve de la pelvis renal, fenómeno que en nuestra opinión es el hallazgo menos específico y aún en debate entre los distintos criterios utilizados para el diagnóstico de compromiso renal según la $\mathrm{ERD}^{6,9}$.

Por otro lado, el CR tiene limitaciones diagnósticas frente a la presencia de lesiones morfológicas, como quistes, hidronefrosis, infartos y lesiones cicatriciales, por lo que, es indispensable combinar este estudio con al menos una ecografía renal tradicional, en la evaluación de una ITU febril2 ${ }^{23}$.

\section{Conclusión}

Dados los resultados del presente estudio y los argumentos discutidos en este artículo, podemos plantear que una ERD sugerente de PNA en un niño hospitalizado por una ITU febril puede confirmar, con bastante seguridad, la presencia de compromiso renal. Ahora bien, si la ERD es negativa y el paciente presenta parámetros inflamatorios alterados, el compromiso renal debe ser confirmado por un $\mathrm{CR}$, ya que existe evidencia de ciertas localizaciones más difíciles de identificar por la ERD, como aquellas que afectan el polo superior izquierdo.

\section{Referencias}

1.- Cavagnaro F: Infección urinaria en la infancia. Rev Chil Infect 2005; 22: 161-8.
2.- $\quad$ Shaikh N, Ewing A, Bhatnagar S, Hoberman A: Risk of renal scarring in children with a first urinary tract infection: A systematic review. Pediatrics 2010; 12: 1084-91.

3.- Evans $J$ : Investigation of urinary tract infection in children. Current Paediatrics 2006; 16: 248-53.

4.- Majd M, Nussbaum A, Markle B, Shalaby-Rana E, et al: Acute pyelonephritis comparison of diagnosis with ${ }^{99 m}$ TC-DMSA, SPECT, spiral CT, MR imaging and power Doppler US in an experimental pig model. Radiology 2001; 218: 101-8.

5.- Halevy R, Smolkin V, Bykov S, Chervinsky L, Sakran W, Koren A: Power Doppler ultrasonography in the diagnosis of acute childhood pyelonephritis. Pediatr Nephrol 2004; 19: 987-91.

6.- Brader P, Riccabona M, Schwarz T, Seebacher U, Ring $E$ : Value of comprehensive renal ultrasound in childre with acute urinary tract infection for assessment of renal involevement: comparison with DMSA scintigraphy and final diagnosis. Eur Radiol 2008; 18: 2981-9.

7.- Donoso G, Lobo G, Arnello F, et al: Cintigrama renal DMSA en niños con primera pielonefritis aguda: correlación con exámenes de laboratorio, ecografía y la presencia de reflujo vésico ureteral. Rev Med Chile 2004; 132: 58-64.

8.- Basiratnia M, Noohi AH, Lotfi M, Alavi MS: Power Doppler sonographic evaluation of acute childhood pyelonephritis. Pediatr Nephrol 2006; 21: 1854-7.

9.- Stoggiani A, Nikolopoulos P, Oikonomou I, et al: Childhood acute pyelonephritis: comparison of power Doppler sonography and Tc-DMSA scintigraphy. Pediatr Radiol 2007; 37: 685-90.

10.- Ochoa C, Málaga S, Panel de expertos de la Conferencia de Consenso y Grupo Investigador de la Conferencia de Consenso: Recomendaciones de la Conferencia de Consenso "Manejo Diagnóstico y Terapéutico de las Infecciones del Tracto Urinario en la Infancia". An Pediatr (Barc) 2007; 67: 517-25.

11.- Mandell GA, Eggli DF, Gilday DL, et al: Society of Nuclear Medicine procedure guideline for renal cortical scintigraphy in children. Society of Nuclear Medicine Procedure Guidelines Manual August 2003; 195-8.

12.- Piepz A, Ham H: Pediatric applications of renal nuclear medicine. Seminars Nucl Med 2006; 36: 16-35.

13.- Comité de Microbiología Clínica, Sociedad Chilena de Infectología: Recomendaciones para el diagnóstico microbiológico de la infección urinaria. Rev Chil Infect 2001; 18: 57-63.

14.- Hitzel A, Liard A, Vera P, Manrique A, Menard JF, Dacher JN: Color and Power Doppler Sonography versus DMSA scintigraphy in acute pyelonephritis and in pre- 
diction of renal scarring. J Nucl Med 2002; 43: 27-32.

15.- Morin D, Veyrac C, Kotzki PO, et al: Comparison of ultrasound and dimercaptosuccinic acid scintigraphy changes in acute pyelonephritis. Pediatr Nephrol 1999; 13: 219-22.

16.- Mohkam M, Maham S, Rahmani A, et al: Ruhollah Ghazi, Majid Ahoopai Technetium Tc 99m Dimercaptosuccinic Acid Renal Scintigraphy in Children With Acute Pyelonephritis Correlation With Other Imaging Tests. IJKD 2010; 4: 297-301.

17.- Garín EH, Olavarría F, Araya C, Broussain M, Barrera $C$, Young $L$ : Diagnostic significance of clinical and laboratory findings to localize site of urinary infection. Pediatr Nephrol 2007; 22: 1002-6.

18.- Huang DT, Huang FY, Tsai TC, Tsai JD, Chiu NC, Lin CC: Clinical differentiation of acute pyelonephritis from lower urinary tract infection in children. J Microbiol Immunol Infect 2007; 40: 513-7.

19.- Fernández-Menéndez JM, Málaga S, Matesanz JL, Solís G, Alonso S, Pérez-Méndez C: Risk factors in the deve- lopment of early technetium-99m dimercaptosuccinic acid renal scintigraphy lesions during first urinary tract infection in children. Acta Pædiatr 2003; 92: 21-6.

20.- Piepz A, Blaufox MD, Gordon I, et al: Consensus on renal cortical scintigraphy in children with urinary tract infection. Scientific Committee of radionuclides in nephrourology. Semin Nucl Med 1999, 29: 160-74.

21.- Montini G, Zucchetta P, Tomasi L, et al: Value of imaging after a first urinary tract infection in Young children: Data from Italian renal infection study 1. Pediatrics 2009; 123: e239-46.

22.- Craig JC, Wheeler DM, Irwig L, Howman-Giles RB: How accurate is dimercaptosuccinic acid scintigraphy for the diagnosis of acute pyelonephritis? A metaanalysis of experimental studies. J Nucl Med 2000; 41: 986-93.

23.- Bjorgvinsson E, Majd M, Eggli KD: Diagnosis of acute pyelonephritis in children: comparison of sonography and 99mTc-DMSA scintigraphy. Am J Roentgenol 1991; 157: 539-43. 